\section{EL IMPACTO DE INTERNET EN LA PARTICIPACIÓN POLÍTICA: REVISANDO EL PAPEL DEL INTERÉS POLÍTICO}

\author{
Rosa Borge \\ Ana Sofía Cardenal \\ Profesoras Agregadas de Ciencia Política. \\ Internet Interdisciplinary Institute (IN3). \\ Universitat Oberta de Catalunya (UOC). Barcelona \\ Claudia Malpica \\ Doctoranda del Departamento de Estadística \\ Universidad de Salamanca
}

\begin{abstract}
In this paper we aim to investigate the relationship between Internet use, motivation and political participation. In particular, we aim to find out if, by reducing participation costs, use of the Internet changes the importance of motivation in the classic explanations of participation. In order to explore this question, we have used data from survey 2736 by the Centro de Investigaciones Sociológicas (CIS), which addresses the issue of political participation and Internet use. The analysis shows that Internet use has a direct impact on participation, regardless of motivation, and that skilled Internet users do not need to be motivated or interested in politics in order to participate in at least one online political activity.
\end{abstract}

KEY WORDS: Online political participation; Internet; Political Interest; Internet Skills.

\section{INTRODUCCIÓN}

El uso de Internet ha generado un rico y controvertido debate sobre sus efectos en la participación política. El nuevo medio tiene unas características muy poderosas que permiten pensar que, directa o indirectamente, puede afectar a la participación política. Por un lado, Internet incrementa en una cantidad sin precedentes el volumen de información en circulación. Aunque aún se debate si este entorno rico en información que crea Internet aumenta o reduce los costes de información (Bimber, 2001, 2003; Anduiza, Gallego y Jorba, 2009), es previsible que, cualesquiera que sean sus efectos sobre la información, tengan consecuencias sobre la participación. Por otro lado, Internet es un medio interactivo que multiplica las posibilidades de contacto de una forma muy eficiente en términos de inversión en tiempo y crea

\section{THE IMPACT OF THE INTERNET ON POLITICAL PARTICIPATION: REVISITING THE ROLE OF POLITICAL INTEREST}

RESUMEN: El objetivo de este artículo es investigar la relación entre el uso de Internet, la motivación y la participación política. En concreto nos interesa descubrir si el uso de Internet cambia la importancia que se da a la motivación política en las explicaciones clásicas de la participación. Para examinar esta cuestión, se ha empleado la encuesta 2736 del Centro de Investigaciones Sociológicas (CIS) que versa sobre participación política y usos de Internet. Los análisis muestran que el uso de Internet tiene un efecto positivo sobre la participación, independientemente del interés por la política, y que los usuarios de Internet que son habilidosos con este medio no necesitan estar motivados o interesados en la política para participar al menos en una actividad política por Internet.

PALABRAS CLAVE: Participación política en línea; Internet; Interés Político; Habilidades con Internet.

un entorno intensivo de comunicación. Asimismo, Internet permite la creación y recreación de "espacios" donde la discusión y la deliberación sobre temas de interés común son posibles (Karakaya, 2005). Finalmente, el uso de Internet ayuda a que las actividades de participación "tradicionales" (como contactar con políticos, firmar una petición, donar dinero, etc.) se puedan desempeñar mucho más fácilmente y reduce ciertos costes de transacción -como la comunicación- implicados en la acción colectiva (Bonchek, 1995).

Estas características de Internet, que le son propias y específicas y que la diferencian de otros medios de comunicación, han llevado a preguntarse si su uso puede afectar a los patrones clásicos de comportamiento en relación con la participación política, alterando los niveles y los estilos de la participación política. Es decir, estas características del 
medio conducen a preguntarse si Internet puede cambiar quién, cómo y por qué se participa en política.

Existen varios estudios ${ }^{1}$ centrados en cómo Internet está cambiando el modelo clásico que explica la participación política a través de los recursos. Este modelo fue definitivamente fijado por Verba, Schlozman y Brady (1995) y ha disfrutado de un amplio reconocimiento dentro de la ciencia política. Sin embargo, pocos estudios ${ }^{2}$ han analizado si Internet puede transformar esta aproximación clásica en cuanto al cambio que puede provocar en la relevancia de la motivación política. Para el modelo tradicional, la implicación psicológica en política o, en otras palabras, la motivación política es un elemento clave necesario para la participación, junto con los recursos consistentes en la disponibilidad de tiempo, dinero y habilidades cívicas (Verba, Schlozman y Brady, 1995: 269). Nuestro argumento es que, a través de la reducción de los costes de la participación, el uso de Internet aumenta la probabilidad de participar en al menos una actividad en línea por parte de usuarios habilidosos y experimentados, incluso en ausencia de motivación política.

El artículo consta de seis apartados. En el primer apartado se revisan las diferentes posiciones que existen en la literatura sobre la capacidad de Internet para atraer a nuevos tipos de participantes y se repasan los pocos estudios que han analizado el papel de la motivación política en la movilización de los participantes en actividades políticas por Internet. En el segundo apartado se compara la perspectiva instrumental o de la elección racional sobre la participación con el modelo clásico basado en los recursos, principalmente respecto al rol de la motivación política. En el tercer apartado se explica cómo el uso de Internet puede afectar al papel que juega la motivación y, posteriormente, se formulan las hipótesis que se comprobarán en este estudio. El cuarto apartado describe los datos y las medidas empleados en el análisis. En el apartado quinto se presentan los resultados del análisis y la correspondiente discusión de los resultados. Por último, el artículo se cierra con las conclusiones.

\section{INTERNET, MOVILIZACIÓN Y MOTIVACIÓN POLITICA: EL DEBATE}

Existen en la literatura diferentes posiciones respecto a la cuestión de si Internet puede cambiar quién, cómo y por qué se participa en política.
Por un lado, están las tesis que sostienen que Internet no sólo no modificará la lógica de la participación, sino que tendrá un efecto negativo en la participación. Apuntada por Robert Putnam en "Bowling Alone" (2000), esta tesis defiende que Internet no propicia la creación de capital social; primero, porque su uso sustituye a las relaciones interpersonales $y$, segundo, porque se usa fundamentalmente para actividades de entretenimiento.

Entre los que sostienen que Internet tendrá un efecto positivo en la participación, se distinguen dos posiciones. Por un lado, están los que sostienen que Internet se limitará fundamentalmente a intensificar la participación de los que ya participan $y$, por tanto, a estos autores se les ha englobado en la denominada tesis de la normalización o del refuerzo. Estos argumentos se sustentan en que, después de un periodo excepcional, el del principio, en que el uso de Internet generó expectativas de cambio en el comportamiento social, la actividad en Internet se ha ido normalizando y los individuos, poco a poco, se han dedicado a hacer a través de este medio lo que ya hacian presencialmente (Margolis y Resnick, 2000). Aplicada a la participación, esta tesis de la normalización ha defendido que Internet, lejos de movilizar a gente nueva que hasta ahora no participaba en política, ha servido de refuerzo a los que ya participaban en política (Norris, 2001; Bimber, 2001).

Por otro lado, están los que defienden la tesis de que Internet no sólo tendrá un efecto positivo en la participación, sino que movilizará a individuos que hasta ahora eran inactivos y no tenían el perfil del participante tradicional. En otras palabras, esta tesis defiende que Internet puede modificar la lógica de la participación a través de la movilización de individuos y grupos nuevos de individuos que hasta ahora se habian mantenido al margen del proceso participativo (Delli Carpini, 2000; Ward, Gibson y Lusoli, 2003)3.

Recientemente, han aumentado las contribuciones que aportan evidencias que apoyan la tesis de la nueva movilización (Krueger, 2002; Tolbert y McNeal, 2003; Quintelier y Vissers, 2008; Cantijoch, 2009; DiGenaro y Dutton, 2006; Gibson, Lusoli y Ward, 2005) ${ }^{4}$. Por ejemplo, Cantijoch (2009) encuentra que el uso de Internet aumenta las actividades no convencionales de participación (como la protesta y las actividades de boicot de productos de consumo) y que este aumento de las formas no convencionales de participación 
se debe no sólo a la participación de individuos críticos que son usuarios de Internet, sino también a la de individuos que tradicionalmente han participado en actividades convencionales y que, por efecto de Internet, ahora participan más en las no convencionales. Otros estudios, como los de Quintelier y Vissers (2008), DiGenaro y Dutton (2006), Gibson, Lusoli y Ward (2005) y Mossberger, Tolbert y McNeal (2008), han destacado que el uso de Internet está movilizando grupos que tradicionalmente han participado con niveles muy inferiores a los de otros colectivos, como los jóvenes y las mujeres.

Los estudios de Best y Krueger (2005), Gibson, Lusoli y Ward (2005) y Anduiza, Gallego y Cantijoch (2010) también respaldan la tesis de la nueva movilización, al destacar que los recursos que dan cuenta de la participación online ya no son sólo los tradicionales, como el tiempo, el dinero y las habilidades cívicas, sino también la habilidad con Internet. Anteriormente, Krueger (2002) sostenía que las habilidades con Internet eran el factor predictivo más importante de la participación en línea, de manera que las habilidades cívicas clásicas no eran significativas 0 incluso los ingresos familiares disminuian la probabilidad de participar en línea (pp. 487-488). Otros autores, como Jensen, Danzinger y Venkatesh (2007), aportan evidencias de la pérdida de peso del estatus socioeconómico para la participación por Internet, al menos en los EE.UU. Finalmente, Gibson, Lusoli y Ward (2005) encuentran que estar sometido a ciertos estímulos a través de la red, como ser contactado, aumenta la probabilidad de la participación online o de entrar en contacto con políticos, partidos y candidatos.

Todos estos estudios aportan pruebas que apoyan la tesis de la nueva movilización, y algunos no sólo aportan evidencias empíricas sino que proponen mecanismos que explican cómo el uso de Internet afecta a la participación. Sin embargo, entre los estudios sobre la nueva movilización, muy pocos se han preguntado si el uso de Internet afecta a la lógica de la participación política al modificar el papel que la motivación tiene en los modelos clásicos de participación. Sabemos, por las explicaciones clásicas, que la predisposición psicológica a participar es un factor tan importante como necesario para dar cuenta de la participación. La participación política es costosa y lo que permite superar estos costes -según las explicaciones clásicas- es, en buena parte, la predisposición psicológica a participar $y$, en otra gran parte, los recursos vinculados al estatus socio-económico, como el tiempo, el dinero y las habilidades cívicas. Hasta ahora, la literatura que investiga el efecto movilizador de Internet sólo ha examinado cómo Internet puede modificar los recursos necesarios para la participación política (Krueger, 2002; Best y Krueger, 2005; Anduiza, Gallego y Cantijoch, 2010; Gibson, Lusoli y Ward, 2001), pero no si y cómo puede modificar el papel que la motivación o la predisposición psicológica a participar ha tenido en las explicaciones clásicas. De hecho, todos o casi todos los estudios más recientes sobre el impacto del uso de Internet en la participación dan por sentada la importancia de la motivación, al incorporar siempre en sus modelos este factor como variable de control. Sin embargo, si aceptamos el argumento utilizado en los estudios sobre la nueva movilización de que Internet reduce los costes de la participación, ¿por qué no preguntarnos si esto tiene algún efecto sobre el papel de la motivación a la hora de dar cuenta de la participación?

Éste no sólo es un tema poco tratado en la literatura, sino que los pocos trabajos que han dicho algo sobre él, aunque sea marginalmente, llegan a resultados contradictorios. Por un Iado, Di Genaro y Dutton (2006), analizando los datos de una encuesta realizada por el Oxford Internet Institute sobre usos de Internet, encuentran que cuando se introduce el uso de Internet en el modelo que explica la participación online, desaparece el efecto de la motivación o el interés por la política. Es decir, que lo que parece tener un efecto independiente y directo en la participación online es el uso de Internet y no la motivación. Xenos y Moy (2007) y Anduiza, Gallego y Jorba (2009) hallan resultados menos contundentes pero que apuntan en la misma dirección. Xenos y Moy (2007) descubren que el uso de Internet por sí mismo (concretamente, exponerse a información sobre la campaña electoral por Internet) aumenta el conocimiento político independientemente de la motivación. En cuanto a Anduiza, Gallego y Cantijoch (2010) encuentran que el efecto conjunto del uso de Internet y la motivación en el conocimiento político es el inverso al esperado: parece que el efecto del uso de Internet en el conocimiento político es mayor entre los individuos no motivados que entre los motivados políticamente.

En cambio, hay trabajos que apuntan exactamente en la dirección opuesta. Por ejemplo, en su meta-análisis, Boulianne (2009) señala que en muchos trabajos que exa- 
minan el efecto del uso de Internet en la participación, este efecto desaparece cuando se introduce la motivación ${ }^{5}$. Según Boulainne (2009), estos resultados podrian estar demostrando no sólo que el uso de Internet no tiene un efecto independiente en la participación, sino que la asociación positiva entre el uso de Internet y la participación se explica por la influencia de la motivación, que sería el factor explicativo clave.

En resumen, pocos trabajos han examinado la cuestión de si Internet puede modificar el papel que la motivación tradicionalmente ha tenido en las explicaciones clásicas sobre participación. Los pocos trabajos que han dicho algo sobre el tema no sólo no contribuyen demasiado a dilucidar la relación que existe entre el uso de Internet, la motivación y la participación, sino que ofrecen resultados contradictorios. No queda claro si el uso de Internet tiene un efecto independiente en la participación que elimina el efecto de la motivación (DiGenaro y Dutton, 2006), si tiene un efecto directo en la participación que es independiente de la motivación (Xenos y Moy, 2007) o, finalmente, si la relación entre el uso de Internet y la participación es espuria y el factor causal de la participación (tanto online como offline) sigue siendo la motivación (Boulianne, 2009; Bimber, 2001).

En este artículo nos proponemos investigar un poco más la relación que existe entre el uso de Internet, la motivación y la participación política. En particular, queremos saber si el uso de Internet, al reducir los costes de la participación, modifica la importancia que la motivación ha tenido en las explicaciones clásicas sobre participación. Para examinar esta cuestión utilizamos datos de la encuesta 2736 del CIS que versa sobre la participación política y los usos de Internet.

\section{¿POR QUÉ PARTICIPAR?: LA PERSPECTIVA INSTRUMENTAL FRENTE AL MODELO CLÁSICO DE LA PARTICIPACIÓN}

Uno de los fenómenos menos comprendido y más difícilmente explicable desde una perspectiva instrumental o de coste-beneficio del comportamiento humano es la participación. La participación es un fenómeno de difícil explicación desde una perspectiva instrumental porque, siendo una actividad costosa, no está claro qué beneficios reporta. La dificultad para entender los beneficios que reporta la participación se deriva, como ya explicaron Downs (1957) y Olson (1965), de las caracteristicas del principal bien que se persigue con la participación: el bien público. Según los formuladores originales de la teoría, los bienes públicos no son un incentivo o una razón suficiente para la participación al menos por dos razones. En primer lugar, porque una vez proveído el bien público, el beneficio es compartido por todos, independientemente de quién haya participado. En segundo lugar, porque la influencia que un individuo aislado tiene en la provisión del bien público es extremadamente pequeña. Cuando se tiene en cuenta que el esfuerzo de un individuo aislado contribuye tan poco al resultado y que, haya o no participado, podrá disfrutar igualmente de los beneficios de la acción colectiva, se entiende que el individuo no tenga incentivos para participar y que quiera aprovecharse del esfuerzo de los demás. Esta lógica es la que lleva a predecir que muy poca gente participará de forma voluntaria en actividades civicas y políticas. Sin embargo, desafiando a esta lógica, se observa que las personas participan voluntariamente en mucha mayor proporción que la esperada por la teoría. De ahí, la llamada "paradoja de la participación".

La utilidad de plantear el problema de la participación desde esta óptica es que nos lleva a hacernos la siguiente pregunta -pregunta que ya se hizo Olson (1965)-: si los beneficios colectivos no son una razón suficiente para explicar la participación, entonces ¿qué otras razones son las que mueven al individuo a participar de forma voluntaria en las actividades cívicas y políticas? Por supuesto, la respuesta de Olson a esta pregunta, y una de sus grandes contribuciones a la ciencia política, es que esas otras razones que mueven al individuo a participar en actividades colectivas son los Ilamados "incentivos selectivos". Puesto que a Olson (1965) le interesaba explicar la decisión de los individuos de unirse a un grupo, entendió los incentivos selectivos como una respuesta/estrategia de las organizaciones dirigida a superar el problema de la acción colectiva. Esta respuesta consistía, según Olson (1965), en el reparto de bienes privados, principalmente materiales, como el disfrute de seguros de salud, del sistema de pensiones, de viajes, etc., como parte de los beneficios de pertenecer a una organización. Junto a los incentivos selectivos positivos (los beneficios materiales), Olson (1965) también destacó el papel de los incentivos selectivos negativos, como la coerción, para explicar por qué las organizaciones gran- 
des sobreviven. Con posterioridad a Olson, los incentivos selectivos fueron utilizados para explicar otras formas de participación como la decisión de votar (Riker y Ordeshook, 1968; Aldrich, 1983) o la decisión de trabajar voluntariamente para un partido político o candidato (Aldrich, 1983; Clark y Wilson, 1961; Whitely et al., 1994; Whitely y Seyd, 1998; Granick, 2005). El problema de utilizar los incentivos selectivos para explicar múltiples y cada vez más variadas formas de participación es que poco a poco el concepto se ha ido ensanchando para convertirse en un cajón de sastre donde han ido a parar todo tipo de razones para participar. De esta forma, nos encontramos que, en explicaciones recientes y no tan recientes sobre distintas formas de participación, los incentivos selectivos incluyen no sólo los beneficios materiales en los que principalmente pensó Olson, sino también beneficios intangibles o inmateriales como la gratificación que se deriva del acto mismo de participar, lo que también se conoce en la literatura como comportamiento expresivo.

El problema, como ya han señalado observadores sagaces como Barry (1978), es que incluir entre los beneficios de una acción la propia acción o el propio comportamiento va en contra de la lógica misma del enfoque instrumental, cuya característica consiste en explicar el comportamiento humano a partir de sus consecuencias y no como una finalidad en sí mismo. Además, la participación, como explican Verba, Schlozman y Brady (1995), presenta un problema adicional para el enfoque instrumental, y es que, en muchos casos, el beneficio o recompensa de la acción de participar se deriva directamente de sus costes. Tras realizar entrevistas a los activistas, Verba, Schlozman y Brady (1995: 103) observan que "un objetivo que se ha conseguido como resultado de una dura lucha se llena de significado, mientras que una victoria fácil a veces parece trivial o, como mínimo, inmerecida" ${ }^{6}$. $Y$ concluyen que "en estas circunstancias, a mayor tiempo, dinero o esfuerzo invertido, mayor es el nivel de gratificación" (Ibídem). En otras palabras, "soportar los costes forma parte de los beneficios" (Ibídem). De aquí se deriva que cuando se trata de la participación no sea tan fácil distinguir entre costes y beneficios $y$, consiguientemente, esto añade un problema más a la capacidad del enfoque instrumental para explicar la participación.

En contraste con el enfoque instrumental, las explicaciones sociológicas no tienen ninguna obligación -para ser coherentes- de distinguir nítidamente entre los costes y beneficios de la participación (aunque igualmente sean explicaciones encubiertas de costes y beneficios) porque su objetivo es estimar cómo los atributos socioeconómicos de los individuos determinan su propensión a participar. Estas explicaciones escapan al problema de que en la participación los costes también pueden ser beneficios mediante la introducción de la motivación en el modelo. Verba, Schlozman y Brady (1995) entienden la motivación como la predisposición psicológica de los individuos a participar en los asuntos públicos. Esta predisposición psicológica a participar es, en realidad, responsable de trasformar una parte de los costes de la participación en beneficios y, por tanto, el solo hecho de tener esta predisposición psicológica o la motivación ya ayuda al individuo a superar una parte de los costes de la participación. La otra parte de los costes de la participación, en las explicaciones sociológicas clásicas, se supera con recursos como el dinero, el tiempo y ciertas habilidades que vienen determinadas por la posición de los individuos en la estructura socioeconómica. La motivación, pues, en las explicaciones sociológicas, es equivalente a la gratificación que se obtiene con la participación voluntaria en actividades cívicas y políticas.

Aunque el factor explicativo clave de la participación en las explicaciones sociológicas no es la motivación sino los recursos, de la obra de Verba, Schlozman y Brady (1995) se deduce que la motivación o la implicación psicológica tienen un papel clave en la participación. De hecho, de una lectura cuidadosa de Verba, Schlozman y Brady (1995) se desprende que el efecto de los recursos dependerá de la implicación psicológica del individuo. Como literalmente señalan estos autores, "los recursos de tiempo, dinero y habilidades civicas facilitan al individuo que ya tiene predisposición a participar ( $y$, añadimos, no al que no la tiene) a hacerlo"7 (Verba, Schlozman y Brady 1995: 334). Dicho de otra forma, por lo que dicen estos autores, parece que el efecto de los recursos sobre la participación no es independiente de la motivación, sino que depende de los valores que adopte esta variable. Esta relación condicionada de la motivación sobre el efecto que los recursos tienen en la participación se confirma en un apartado del apéndice D del mismo libro (Verba, Schlozman y Brady 1995: 609) donde se admite que: 1) el efecto conjunto o multiplicativo de estas variables (motivación medida como interés político y recursos medidos como habilidades cívicas) es positivo y significativo, y 2) que al introducir el término de la interac- 
ción, uno de los efectos específicos de estas variables sobre la participación desaparece, aunque los autores no explican a qué variable se están refiriendo. Dicho de otra manera, y teniendo presente que no sabemos qué variable es la que ha perdido la significatividad, parece que una cierta predisposición a participar aumenta la propensión a participar, la cual también está influida por los recursos (tener tiempo, dinero y habilidades) de que dispone el individuo.

Por supuesto, todo esto tiene perfecto sentido cuando tenemos en cuenta que participar es costoso. Precisamente porque participar es costoso es necesaria una explicación que no sólo ponga el acento en los recursos, sino también en la motivación. Una explicación sólo basada en los recursos puede aspirar a explicar quién no participa en política, pero no quién participa. Para explicar quién participa en política, la motivación, entendida como un factor de reducción de costes adicional que opera transformando los costes de la participación en beneficios, parece ser un factor fundamental.

\section{INTERNET, MOTIVACIÓN POLÍTICA Y PARTICIPACIÓN: ¿Cómo Se Relacionan?}

Como deciamos al principio, el uso de Internet ha generado un rico debate sobre sus posibles efectos en la participación. Una de las premisas que subyace detrás de este debate y en la que coinciden muchas de las voces que han participado en él es que Internet reduce los costes de la participación. Por un lado, se ha destacado que Internet reduce los costes de estar informado al multiplicar el volumen de información en circulación y permitir el acceso a fuentes diversas de información. Es verdad que, aunque se pueda argumentar que Internet hace más fácil el acceso a la información, esto no necesariamente reduce los costes de procesamiento de esta información, que seguirian siendo altos o incluso podrían ser más altos debido al aumento de opciones de información que supone Internet. Es decir, que aunque Internet aumenta las opciones de información, esto no se traduce necesariamente en más conocimiento político y/o en participación (Bimber, 2001; Anduiza, Gallego y Jorba, 2009).

Por otro lado, al ser un medio interactivo en el que cabe la comunicación bilateral, de uno a muchos y de muchos a muchos, Internet ofrece unas posibilidades enormes de contacto y comunicación y reduce mucho los costes para establecer contactos. Este entorno intensivo en comunicación que crea Internet puede reducir los costes $-y$, por tanto, afectar- tanto a la participación offline como a la participación online. En lo que afecta a la participación offline, Internet puede ayudar a reducir determinados costes de transacción, especialmente los costes de información y comunicación, que están presentes en la formación y acción de los grupos. Como dice Bonchek (1995), al reducir los costes de información y comunicación, Internet reduce los costes de coordinación que están implicados en la acción colectiva y, así, facilita la acción de los grupos. Internet también puede estimular la participación offline y reducir sus costes porque abarata también los costes de las campañas de movilización de las organizaciones políticas. Gracias a Internet es posible que estas campañas de movilización lleguen a un público potencial mucho más amplio con un coste muchísimo más bajo ${ }^{8}$.

Pero donde Internet ofrece las mayores ventajas es en la participación online. La facilidad de contacto y de comunicación a través de la red permite realizar las actividades tradicionales de participación que hasta ahora eran relativamente costosas, como contactar con políticos, colaborar con organizaciones, afiliarse a un partido, colaborar en campañas y/o participar en foros y grupos de discusión, a un coste bajísimo. De hecho, para recibir información o suscribirse a algún boletín, contactar con algún político, hacer alguna donación, formular una queja a la administración, contactar con una asociación, colaborar con una campaña, participar en algún foro de discusión, etc..., basta con estar conectado y sin necesidad de moverse del sillón hacer un click en el teclado del ordenador. En pocas palabras, el uso de Internet, al permitir llevar a cabo a través de la red actividades tradicionales de participación como las mencionadas, ha reducido hasta un punto insospechado el coste de realizar tales actividades.

Lo que aquí argumentamos y trataremos de probar es qué, al reducir tan drásticamente los costes de la participación, el uso de Internet puede llegar a modificar el papel que la motivación ha tenido en las explicaciones clásicas -las sociológicas- sobre la participación. ¿Cómo? Pues modificando la relación entre los recursos y la participación que en los modelos clásicos de participación estaba condicionada por la motivación. La idea es que 
cuando los costes de la participación son suficientemente bajos, puede ocurrir que tener los recursos necesarios, en este caso tener los recursos de Internet, sea un factor suficiente para explicar la participación. Esta idea ya la encontramos en un artículo de Xenos y Moy (2007), en donde los autores muestran que buscar a través de Internet información sobre la campaña tiene un efecto sobre el conocimiento político independiente de la motivación o del interés por la política (p. 713), y también prueban que el interés político no afecta a la exposición a la información política a través de Internet (p. 711). Xenos y Moy (2007) Ilevan a cabo este análisis con el fin de testar la validez de dos enfoques, el instrumental y el psicológico, para explicar la participación, y este descubrimiento es para ellos la confirmación de que el enfoque instrumental es más apto que el enfoque psicológico, al menos para explicar la adquisición de información y de conocimiento político. Aunque es muy discutible lo que estos autores interpretan por ambos enfoques, no se equivocan al asumir que ceteris paribus, desde un enfoque instrumental, una reducción de costes tendrá un efecto directo sobre la participación al aumentar automáticamente la utilidad de la acción. Lo que no tienen en cuenta estos autores es que dependiendo de la actividad $-y$, en concreto, para actividades de muy bajo beneficio- el enfoque instrumental será muy poco apto para hacer predicciones de ningún tipo. Es decir, para acciones con costes y beneficios por debajo de cierto umbral, como el voto y la participación online, la elección racional tendrá muy poco que decir sobre qué gobernará un determinado comportamiento. En estos casos, como explica Aldrich (1983), cualquier cosa que ocurra en el margen podrá tener un efecto significativo en el resultado.

Esta discusión nos lleva a formular dos proposiciones básicas respecto al impacto que el uso de Internet tendrá en la participación, concretamente en la participación online. En el presente artículo intentaremos testar estas dos proposiciones. Ambas se derivan del hecho de que participar por Internet se puede considerar una actividad de bajo coste y bajo beneficio. En primer lugar, al reducir drásticamente los costes de la participación, esperaremos que Internet modifique el papel que la motivación ha tenido en las explicaciones clásicas sobre participación. No esperamos que el uso de Internet elimine el efecto de la motivación sobre la participación, pero sí que tenga un efecto directo en la participación independientemente de la motivación.
Esto no nos permitirá concluir que la motivación deja de ser un factor importante para explicar los diferentes niveles de participación tanto online como offline, pero si que deja de ser un factor necesario para explicar los niveles de participación de los usuarios frecuentes de Internet. De hecho, lo que decimos es que basta con ser un usuario habilidoso con Internet para que aumente la probabilidad de participar en política dentro de la red, independientemente de la motivación. Por tanto, nuestra primera hipótesis la formularemos así:

H1. El uso de Internet no hará desaparecer el efecto principal de la motivación sobre la participación, pero tener habilidades con Internet tendrá un efecto positivo en la participación, independientemente de la motivación.

En segundo lugar, teniendo en cuenta que participar por Internet se puede considerar una actividad de bajo coste y bajo beneficio, incluso por debajo de la acción de votar, esperaremos que, dado un cierto uso de Internet, cualquier pequeño movimiento en el margen, como por ejemplo ser contactado, pueda afectar la probabilidad de participar. Por supuesto, la probabilidad de que se produzca algún movimiento en el margen, como por ejemplo ser contactado, aumentará con el tiempo de exposición en Internet; pero la probabilidad de respuesta dependerá no sólo del tiempo de exposición en Internet, sino también de cómo se navega por Internet; es decir, de si se navega con o sin rumbo fijo. Cuanto más tiempo de exposición por Internet y más errático sea el rumbo, más probable será que haya una respuesta a cualquier movimiento en el margen. Por tanto, esperaremos que tanto navegar sin objetivo fijo por Internet como ser contactado a través de Internet afecten la probabilidad de participar online. $Y$ nuestra segunda hipótesis la formularemos asi:

H2. Además del efecto positivo que tener habilidades con Internet tendrá en la participación online, navegar sin objetivo fijo por Internet y ser contactado por Internet aumentará la probabilidad de la participación online.

\section{DAtos Y MEDICIONES}

Exploraremos la relación entre el uso de internet, la motivación política y la participación en línea a través de

ARBOR Vol. 188756 julio-agosto [2012] 733-750 ISSN: 0210-1963

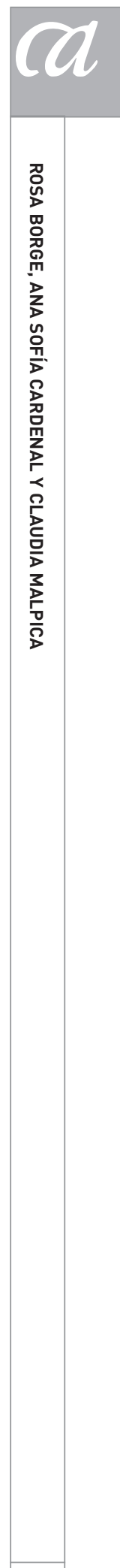

739 
una encuesta representativa de España, administrada por el CIS en noviembre de $2007^{\circ}$. Esta encuesta fue diseñada con el propósito de testar las relaciones entre Internet y la participación política. Por tanto, contiene las preguntas clásicas que se suelen emplear para comprobar el impacto sobre la participación política de las características sociodemográficas, actitudes y orientaciones politicas, y de la exposición a los medios de comunicación. Asimismo, hay preguntas sobre el acceso y los usos de Internet y sobre varias formas de participación en línea. Esta encuesta ha sido ya muy analizada por el equipo de POLNET, cuyas investigadoras han publicado varios artículos y papers utilizando los datos de la encuesta ${ }^{10}$. En consecuencia, no mostraremos otra vez cuánta participación online hay en España, o los modos de las diferentes formas de participación, o los perfiles de los participantes ${ }^{11}$, sino que dirigiremos nuestro trabajo estrictamente a analizar los efectos de la motivación y de las habilidades de Internet sobre la participación online. Nos interesa saber si las habilidades con Internet están teniendo un impacto sobre la participación independiente del nivel de motivación política. Además, queremos averiguar cuál es el rol de ser contactado via email y de navegar por Internet sin un fin concreto a la hora de participar en política por Internet, ya que estos dos factores pueden debilitar la relevancia de la motivación política para la participación online.
En el cuestionario hay hasta 8 actividades políticas desarrolladas por Internet que pueden ser consideradas como participación en política. Hemos seleccionado cinco de ellas tras llevar a cabo varios tests de fiabilidad y dimensionalidad ${ }^{12}$. Por tanto, las cinco actividades pertenecen al mismo constructo y son las siguientes: contactar con un político o partido político; realizar una donación de dinero para una campaña o asociación; escribir comentarios en un foro, blog o página web sobre temas de actualidad, sociales o políticos; firmar una petición o adherirse a alguna campaña o manifiesto; y consultar la página web de un partido político o candidato. De cara al análisis, las cinco actividades fueron examinadas tanto en forma de indice sumatorio de actividades, que va de 0 a 5 actividades, como en forma de variable dummy ( 0 actividades o, como mínimo, una actividad). El propósito de analizar estas dos medidas alternativas de la variable dependiente es testar si los efectos de las variables claves de nuestras hipótesis difieren según el nivel de participación. Es decir, esperamos que las habilidades con Internet tengan un impacto mayor y más directo en la realización de, al menos, una actividad participativa que en la acumulación de un cierto número de actividades. Este último proceso es mucho más exigente y costoso y, por ello, es lógico que los tradicionales recursos y las motivaciones políticas tengan mayor peso.

Respecto a las variables explicativas, se han incluido en nuestro modelo cinco bloques de variables.

TABLA 1. BLOQUES DE VARIABLES EXPLICATIVAS

\begin{tabular}{|c|c|c|c|c|}
\hline $\begin{array}{c}\text { Bloque } 1 \\
\text { Socio- } \\
\text { demográficas }\end{array}$ & $\begin{array}{c}\text { Bloque } 2 \\
\text { Actitudes políticas } \\
\text { y orientaciones }\end{array}$ & $\begin{array}{c}\text { Bloque } 3 \\
\text { Información política a } \\
\text { través de diferentes medios }\end{array}$ & $\begin{array}{c}\text { Bloque } 4 \\
\text { Habilidades con Internet. } \\
\text { Interés político }\end{array}$ & $\begin{array}{c}\text { Bloque } 5 \\
\text { Mecanismos causales }\end{array}$ \\
\hline Nivel educativo & Eficacia interna & $\begin{array}{l}\text { Adquirir información sobre la } \\
\text { actualidad política a través de } \\
\text { Internet }\end{array}$ & Interés político & $\begin{array}{l}\text { Navegar por Internet sin obje- } \\
\text { tivo concreto }\end{array}$ \\
\hline Situación laboral & Eficacia externa & $\begin{array}{l}\text { Escuchar o ver las noticias } \\
\text { (radio o TV) }\end{array}$ & Habilidades con Internet & Ser contactado por email \\
\hline Género & $\begin{array}{l}\text { Confianza en las instituciones } \\
\text { políticas }\end{array}$ & $\begin{array}{l}\text { Escuchar o ver otros programas } \\
\text { sobre politica (radio o TV) }\end{array}$ & $\begin{array}{l}\text { Término de interacción: } \\
\text { Interés político por habilida- } \\
\text { des con Internet }\end{array}$ & \\
\hline Ingresos & $\begin{array}{l}\text { Concepción del deber ciuda- } \\
\text { dano }\end{array}$ & $\begin{array}{l}\text { Leer el periódico (en papel o } \\
\text { por Internet) }\end{array}$ & & \\
\hline Edad & $\begin{array}{l}\text { Concepción de la implicación } \\
\text { de un buen ciudadano }\end{array}$ & & & \\
\hline
\end{tabular}


El cuarto bloque contiene las dos variables explicativas principales de nuestro modelo: el interés político (en cuanto que principal indicador de motivación política) y las habilidades con Internet. La variable interés político dispone de cuatro niveles de interés (nada, poco, bastante, mucho) y las habilidades con Internet son medidas por un proxy formado por un índice de seis actividades online no políti$\operatorname{cas}^{13}$. En este bloque, el interés político se sitúa junto con las habilidades por Internet porque tenemos la intención de explorar sus efectos conjuntos e independientes sobre la participación. Así, en este cuarto bloque se añade el término de interacción entre el interés político y las habilidades por Internet para comprobar si las dos variables producen un efecto combinado sobre la participación online que refuerce su posible impacto individual.

En el quinto bloque se incluyen dos factores que pueden afectar a la probabilidad de participar en línea sin que importe el nivel de interés por la política. La intención es comprobar si navegar por Internet sin objetivo y ser contactado por email incrementa la probabilidad de participar en línea independientemente del nivel de interés político. Si se descubre que las habilidades con Internet incrementan la probabilidad de participar online con independencia de la motivación política, posteriormente deberíamos identificar los mecanismos causales a través de los cuales ser un usuario habilidoso con Internet afecta a la participación. Los dos mecanismos causales (establecidos en la segunda hipótesis) que proponemos son, como ya hemos dicho, navegar por Internet sin objetivo y ser contactado por correo electrónico.

\section{ANÁLISIS Y DISCUSIÓN}

Estudiaremos el efecto de todas las variables mencionadas empleando análisis jerárquicos de regresión múltiple y logística. Cada bloque de variables será introducido de manera separada del resto con la intención de comprobar los efectos y poder clarificador de las principales explicaciones de la participación política basadas en el modelo de los recursos: características socio-demográficas, actitudes políticas y medios a través de los cuales se obtiene la información política. Pero principalmente nuestro interés se centra en comprobar cómo la irrupción de Internet está cambiando el modelo tradicional de la participación.
Así, en el cuarto y quinto bloque se recogen algunas novedades que pueden afectar al modelo clásico debido a la extensión de Internet. En el cuarto bloque se incluye el posible nuevo efecto de los recursos o habilidades con

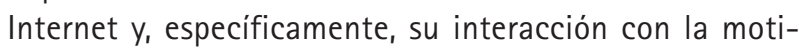
vación política. En el quinto bloque se introduce el efecto de navegar por Internet sin un objetivo concreto, porque puede resultar ser una posible explicación de por qué los internautas acaban expuestos a iniciativas políticas sin planificarlo o sin estar motivados para participar en ellas. En este último bloque también se incluye el efecto de ser contactado por email, ya que puede ser otra posible explicación de por qué los usuarios de Internet, sin planificarlo y sin estar políticamente motivados, acaban participando en actividades políticas en línea ${ }^{14}$. En resumen, el principal objetivo es descubrir los efectos principales e indirectos de las habilidades con Internet y del interés político (hipótesis 1), así como los efectos directos de navegar sin rumbo fijo por Internet y de ser contactado por correo electrónico para realizar actividades políticas (hipótesis 2), controlando por cada uno otro bloque de variables también influyentes.

Las variables que no son significativas en todas las diferentes tandas de ejecuciones de los dos tipos de regresiones son excluidas del análisis. Son las siguientes: las situaciones laborales de estar retirado, ser pensionista o estar parado (del primer bloque), la eficacia externa (también del primer bloque), la concepción del deber ciudadano (del segundo bloque) y leer periódicos en papel o en Internet (del tercer bloque).

Los resultados de los cinco modelos de regresión son presentados en las tablas 2 (regresión múltiple), 3 (regresión logística) y 4 (comparación entre los dos tipos de regresiones). Los dos procedimientos corresponden a las dos mediciones diferentes de la variable dependiente: en la regresión múltiple la variable dependiente es el índice aditivo de actividades (0-5) y en la regresión logística es 0 actividades 0 al menos una actividad.

En las tablas 2 y 3 se puede observar la progresión en capacidad explicativa de los diferentes bloques de variables introducidos. El incremento de capacidad explicativa es continuo $y$, lógicamente, el modelo final que incluye todas las variables explica mejor las variables dependientes.

ARBOR Vol. 188756 julio-agosto [2012] 733-750 ISSN: 0210-1963

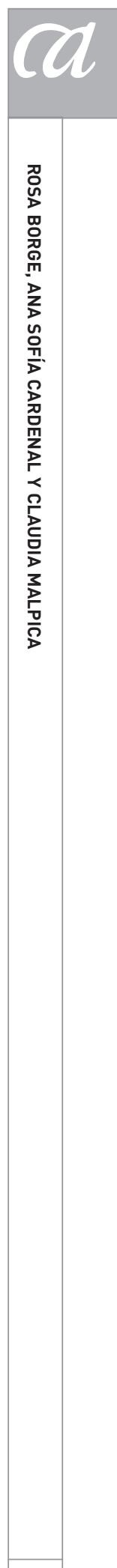

741 
TABLA 2. REGRESIÓN LINEAL MÚLTIPLE DEL ÍNDICE DE PARTICIPACIÓN ONLINE (0-5) SEGÚN LOS 4 BLOQUES DE VARIABLES EXPLICATIVAS

\begin{tabular}{|c|c|c|c|c|c|}
\hline & Bloque 1 & $\mathrm{~B} 1+\mathrm{B} 2$ & $\mathrm{~B} 1+\mathrm{B} 2+\mathrm{B} 3$ & $\mathrm{~B} 1+\mathrm{B} 2+\mathrm{B} 3+\mathrm{B} 4$ & $\mathrm{~B} 1+\mathrm{B} 2+\mathrm{B} 3+\mathrm{B} 4+\mathrm{B} 5$ \\
\hline Nivel educativo & $0.328^{* * *}$ & $0.204^{* * *}$ & $0.112^{* *}$ & 0.077 & 0.045 \\
\hline Estudiante & $0.323^{* * *}$ & $0.225^{* *}$ & $0.207^{* *}$ & $0.258^{* * *}$ & $0.213^{* * *}$ \\
\hline Trabajo doméstico no remunerado & $-0.276^{* * *}$ & $-0.251^{* * *}$ & $-0.177^{* *}$ & $-0.160^{* *}$ & $-0.158^{* *}$ \\
\hline Hombre & $0.0953^{*}$ & 0.0681 & -0.017 & -0.019 & -0.0171 \\
\hline Ingresos & $0.125^{* * *}$ & $0.100^{* * *}$ & $0.091^{* * *}$ & $0.059^{* *}$ & $0.0496^{* *}$ \\
\hline Edad & 0.00414 & -0.00346 & $-0.0055^{*}$ & -0.0028 & -0.0005 \\
\hline Eficacia interna & & $0.286^{* * *}$ & $0.157^{* * *}$ & $0.083^{* *}$ & $0.078^{* * *}$ \\
\hline Confianza en instituciones políticas & & $0.0529^{* * *}$ & $0.037^{* * *}$ & 0.020 & 0.018 \\
\hline Concepción de la implicación de un buen ciudadano & & $0.0366^{* *}$ & $0.029^{* *}$ & 0.020 & 0.017 \\
\hline Información política por Internet & & & $0.242^{* * *}$ & $0.183^{* * *}$ & $0.162^{* * *}$ \\
\hline Escuchar y ver las noticias (radio o TV) & & & $-0.051^{* *}$ & $-0.049^{*}$ & -0.0403 \\
\hline Escuchar y ver otros programas sobre política (radio o TV) & & & $0.055^{* *}$ & 0.0235 & 0.023 \\
\hline Interés político & & & & $0.1995^{* * *}$ & $0.150^{* * *}$ \\
\hline Habilidades con Internet & & & & $0.1202^{* * *}$ & $0.0687^{* * *}$ \\
\hline Interés*Habilidades (centrado) & & & & $0.0775^{* * *}$ & $0.0755^{* * *}$ \\
\hline Navegar sin objetivo & & & & & $0.149^{* * *}$ \\
\hline Ser contactado por email & & & & & $0.525^{* * *}$ \\
\hline _cons & $-1.016^{* * *}$ & $-1.605^{* * *}$ & $-1.168^{* * *}$ & $-1.251^{* * *}$ & $-1.121^{* * *}$ \\
\hline $\mathrm{F}$ & 27.09 & 24.01 & 31.02 & 34.63 & 38.23 \\
\hline Prob $>F$ & 0.0000 & 0.0000 & 0.0000 & 0.0000 & 0.0000 \\
\hline R-cuadrado & 0.0871 & 0.1590 & 0.2747 & 0.3325 & 0.3821 \\
\hline N & 2110 & 1855 & 1834 & 1801 & 1736 \\
\hline
\end{tabular}

Sólo se muestran los coeficientes de regresión y su significatividad: $* p<0.10, * * p<0.05, * * * p<0.01$.

En ambos tipos de regresión la inclusión de las variables de habilidades con Internet, interés político y el término de interacción (los cuales conforman el cuarto bloque) cambia sustancialmente el peso de muchas de las variables sociodemográficas y actitudinales, y también de la adquisición de información política a través de los medios tradicionales. Es decir, muchas de estas variables pierden significatividad, lo cual prueba la importancia de las habilidades con Internet y del interés político sobre muchas de las otras variables a la hora de explicar la participación en línea. Este hecho es incluso más relevante en la explicación de la participación de al menos una actividad (véase la regresión logística en la tabla 3): cuando son incluidas en el modelo las variables de disponer de habilidades con Internet, interés político y el término de interacción, así como navegar sin objetivo concreto y ser contactado por email, únicamente ser estudiante se mantiene como un factor influyente entre las características sociodemográficas. Asimismo, la otra única variable que es significativa en ambos tipos de regresión es usar Internet para obtener información acerca de la actualidad política.

Respecto a la comprobación de las hipótesis, los resultados de los análisis, en primer lugar, confirman la hipótesis 1 tal y como se muestra en la comparación de ambos modelos de regresión (véase tabla 4 dos páginas más adelante).

En el caso del índice de actividades de participación, el efecto del término interactivo es significativo y positivo, así como el efecto específico del interés político y las habilidades con Internet (segunda columna, tabla 4). 
TABLA 3. REGRESIÓN LOGISSIICA DE LA PARTICIPACIÓN ONLINE (0-1) POR LOS CUATRO BLOQUES DE VARIABLES EXPLICATIVAS

\begin{tabular}{|c|c|c|c|c|c|}
\hline & Bloque 1 & $\mathrm{~B} 1+\mathrm{B} 2$ & $\mathrm{~B} 1+\mathrm{B} 2+\mathrm{B} 3$ & $\mathrm{~B} 1+\mathrm{B} 2+\mathrm{B} 3+\mathrm{B} 4$ & $\mathrm{~B} 1+\mathrm{B} 2+\mathrm{B} 3+\mathrm{B} 4+\mathrm{B} 5$ \\
\hline Nivel educativo & $0.674^{* * *}$ & $0.481^{* * *}$ & $0.327^{* *}$ & .217 & 0.108 \\
\hline Estudiante & $0.683^{* * *}$ & $0.509^{* * *}$ & $0.539^{* * *}$ & $.718^{* * *}$ & $0.611^{* * *}$ \\
\hline Trabajo doméstico no remunerado & -0.675 & -0.524 & -0.382 & -.395 & -0.456 \\
\hline Hombre & $0.322^{* * *}$ & $0.272^{* *}$ & 0.1054 & .0537 & 0.0732 \\
\hline Ingresos & $0.215^{* * *}$ & $0.176^{* * *}$ & $0.1710^{* * *}$ & $.0998^{* *}$ & 0.0718 \\
\hline Edad & -0.00141 & $-0.0148^{* *}$ & $-0.021^{* * *}$ & -.0126 & -0.0057 \\
\hline Eficacia interna & & $0.502^{* * *}$ & $0.276^{* * *}$ & .0836 & 0.0638 \\
\hline Confianza en instituciones politicas & & $0.122^{* * *}$ & $0.102^{* * *}$ & $.0679^{*}$ & 0.0645 \\
\hline Concepción de la implicación de un buen ciudadano & & $0.0591^{*}$ & 0.043 & .0237 & 0.0171 \\
\hline Información política por Internet & & & $0.465^{* * *}$ & $.345^{* * *}$ & $0.316^{* * *}$ \\
\hline Escuchar y ver las noticias (radio o TV) & & & -0.0499 & -.0525 & -0.0265 \\
\hline Escuchar y ver otros programas sobre política (radio o TV) & & & $0.0105^{* *}$ & .0267 & 0.0323 \\
\hline Interés político & & & & $.562^{* * *}$ & $0.439^{* * *}$ \\
\hline Habilidades con Internet & & & & $.4006^{* * *}$ & $0.292^{* * *}$ \\
\hline Interés*Habilidades (centrado) & & & & -.0133 & -0.0205 \\
\hline Navegar sin objetivo & & & & & $0.2802^{* *}$ \\
\hline Ser contactado por email & & & & & $1.510^{* * *}$ \\
\hline _cons & $-3.504^{* * *}$ & $-4.714^{* * *}$ & $-4.252^{* * *}$ & $-4.983^{* * *}$ & $-5.0845^{* * *}$ \\
\hline Wald chi2(17) & 93.72 & 127.88 & 219.42 & 271.46 & 350.09 \\
\hline Prob > chi2 & 0.0000 & 0.0000 & 0.0000 & 0.0000 & 0.0000 \\
\hline Pseudo R2 & 0.0612 & 0.1016 & 0.1767 & 0.2278 & 0.2877 \\
\hline$N$ & 2110 & 1855 & 1834 & 1801 & 1736 \\
\hline
\end{tabular}

Sólo se muestran los coeficientes de regresión y su significatividad: $* p<0.10, * * p<0.05, * * * p<0.01$.

Esto significa, por una parte, que el número de actividades participativas aumenta cuando se produce un incremento combinado de las habilidades con Internet y del interés político $y$, en ese sentido, las habilidades con Internet refuerzan el efecto del interés político sobre la participación. Por otra parte, el interés político y las habilidades con Internet influyen separadamente en el número de actividades participativas realizadas. En el caso de tomar parte al menos en una actividad o no participar en nada, el término de interacción no es significativo, pero el interés político y las habilidades por Internet continúan siendo significativos (tercera columna, tabla 4). Este resultado implica que las habilidades con Internet no están intensificando el impacto del interés político sobre la participación. No hay, por tanto, un efecto combinado de ambas variables sobre la participación, y el interés político y las habilidades con Internet desarrollan un efecto específico e independiente sobre la participación en, al menos, una actividad. Las diferencias en significatividad del término de interacción y el efecto específico significativo de las habilidades con Internet en ambos modelos de regresión muestran que, tal como se esperaba, las habilidades con Internet tienen un impacto más directo, independiente del nivel de interés político, sobre la participación en, al menos, una actividad que en la acumulación de un número de actividades. Este último proceso requiere más recursos y predisposiciones actitudinales, tal y como confirma la significatividad que varias variables socio-demográficas, como los ingresos, y actitudinales, como la eficacia interna, mantienen en el modelo de regresión múltiple en contraste con el modelo logístico.

ARBOR Vol. 188756 julio-agosto [2012] 733-750 ISSN: 0210-1963 
TABLA 4. REGRESIONES MÚLTIPLES Y LOGISTICAS COMPARADAS (TODAS LAS VARIABLES EXPLICATIVAS)

\begin{tabular}{|c|c|c|}
\hline & $\begin{array}{l}\text { Núm. de actividades } \\
\text { online }(0-5)\end{array}$ & $\begin{array}{c}\text { Dummy } \\
\text { (0 o al menos } 1 \text { actividad en línea) }\end{array}$ \\
\hline Nivel educativo & 0.045 & 0.108 \\
\hline Estudiante & $0.213^{* * *}$ & $0.611^{* * *}$ \\
\hline Trabajo doméstico no remunerado & $-0.158^{* *}$ & -0.456 \\
\hline Hombre & -0.0171 & 0.0732 \\
\hline Ingresos & $0.0496^{* *}$ & 0.0718 \\
\hline Edad & -0.0005 & -0.0057 \\
\hline Eficacia interna & $0.078^{* * *}$ & 0.0638 \\
\hline Confianza en instituciones políticas & 0.018 & 0.0645 \\
\hline Concepción de la implicación de un buen ciudadano & 0.017 & 0.0171 \\
\hline Información política por Internet & $0.162^{* * *}$ & $0.316^{* * *}$ \\
\hline Escuchar y ver las noticias (radio o TV) & -0.0403 & -0.0265 \\
\hline Escuchar y ver otros programas sobre política (radio o TV) & 0.023 & 0.0323 \\
\hline Interés político & $0.150^{* * *}$ & $0.439^{* * *}$ \\
\hline Habilidades con Internet & $0.0687^{* * *}$ & $0.292^{* * *}$ \\
\hline Interés*Habilidades (centrado) & $0.0755^{* * *}$ & -0.0205 \\
\hline Navegar sin objetivo & $0.149^{* * *}$ & $0.2802^{* *}$ \\
\hline Ser contactado por email & $0.525^{* * *}$ & $1.510^{* * *}$ \\
\hline _cons & $-1.121^{* * *}$ & $-5.0845^{* * *}$ \\
\hline F o Wald chi2(17) & 38.23 & 350.09 \\
\hline Prob $>$ F o Prob $>$ chi2 & 0.0000 & 0.0000 \\
\hline R-cuadrado o Pseudo R2 & 0.3821 & 0.2877 \\
\hline $\mathrm{N}$ & 1736 & 1736 \\
\hline
\end{tabular}

Sólo se muestran los coeficientes de regresión y su significatividad: $* p<0.10, * * p<0.05, * * * p<0.01$.

En conclusión, podemos confirmar la primera hipótesis que afirmaba que disponer de habilidades con Internet no produciria la desaparición del efecto del interés político sobre la participación, pero que tener habilidades con Internet desencadenaría un efecto directo sobre la participación, independientemente del interés político. Por tanto, éste es un indicador de que Internet está teniendo un impacto sustancial en la implicación en política que va más allá del mero refuerzo de los perfiles ya existentes de participantes presenciales. Implica que nuevos tipos de participantes -internautas habilidosos carentes de motivación política- están comenzando a tomar parte en, al menos, una actividad online.

Para una mejor comprensión de la relación entre la variable dependiente y las dos variables de la interacción, se muestran a continuación dos gráficos con las probabilidades de participación según los niveles de interés político y habilidades con Internet. Esto es, se representan los niveles de la variable dependiente según los niveles combinados de interés político y habilidades con Internet. En la figura 1, se muestra la probabilidad de participar en una acción o más por parte de personas sin interés por la política y por parte de personas que están muy interesadas por la política en función de su nivel de habilidades con Internet ${ }^{15}$.

Como se puede apreciar en el primer gráfico, es más probabe que los muy interesados por la política participen en, al menos, una acción política que los nada interesados. Pero además, cuando aumentan las habilidades con Internet no sólo los muy interesados por la política participan más, 
FIGURA 1. PROBABILIDAD DE UNA O MÁS ACCIONES ONLINE SEGÚN EL INTERÉS POLITICO Y LAS HABILIDADES CON INTERNET

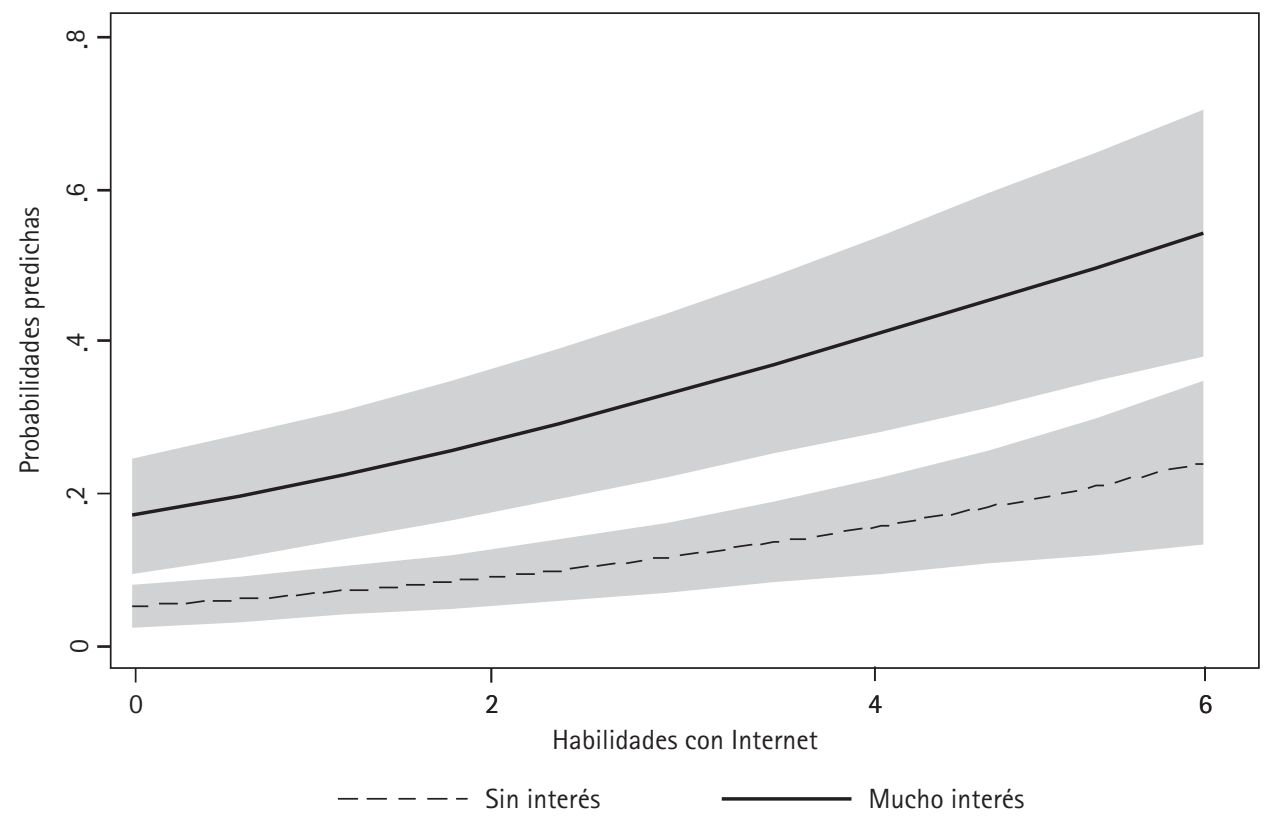

sino también los que no muestran ningún interés por la política. De todas formas, el apuntamiento de las curvas y los valores de las probabilidades muestran que entre los más interesados se produce un mayor incremento de la probabilidad de participar (de 0,3715 puntos) que entre los nada interesados (de 0,18 ).

En la figura 2 de la siguiente página se muestra la misma relación para el mismo perfil, pero situando en el eje horizontal los niveles de interés. Lo que este gráfico muestra más claramente es la importancia de las habilidades con Internet independientemente del nivel de interés político. Así, en este gráfico se indica que entre los que sólo realizan una actividad no política online (no habilidosos), el incremento del interés político no cambia de forma importante las probabilidades de participar, que siguen siendo muy bajas. El apuntamiento de esta curva es pequeño en comparación con la curva de los muy habilidosos, es decir, los que llegan a acumular hasta seis actividades por Internet. Asimismo, se observa que un hombre muy interesado en política, pero que sólo dispone de una habilidad con Internet, tiene algo menos de probabilidad $(0,22)$ de participar online que alguien no interesado, pero muy habilidoso con
Internet, que realiza hasta seis actividades no políticas online $(0,24)$. Por tanto, los internautas muy habilidosos participan más en política que los poco habilidosos y su probabilidad de participar se incrementa de forma importante a medida que aumenta su interés por la política $(0,30)$. No obstante, cuando no se tienen habilidades con Internet, la probabilidad de participar no depende tanto del nivel de interés político, ya que la probabilidad de participar aumenta poco con el aumento del interés por la política (sólo 0,15 puntos).

Tras todos estos análisis en los que se comprueba que el impacto de las habilidades sobre la participación es muy importante e independiente de la motivación política, debemos asegurarnos de que dicho impacto no es espurio, siendo la motivación la verdadera causa (Boulainne, 2009; Bimber, 2001). Es decir, puede ocurrir que los usuarios de Internet habilidosos estén concentrados entre los más interesados por la política. Para examinar esta posibilidad se ha llevado a cabo una regresión múltiple de la variable de habilidades con Internet por el resto de variables (incluyendo interés político $\left.{ }^{16}\right)$, que se recoge en la tabla 5 .

ARBOR Vol. 188756 julio-agosto [2012] 733-750 ISSN: 0210-1963 
FIGURA 2. PROBABILIDAD DE UNA O MÁS ACCIONES ONLINE SEGÚN LAS HABILIDADES CON INTERNET Y EL INTERÉS POLITICO

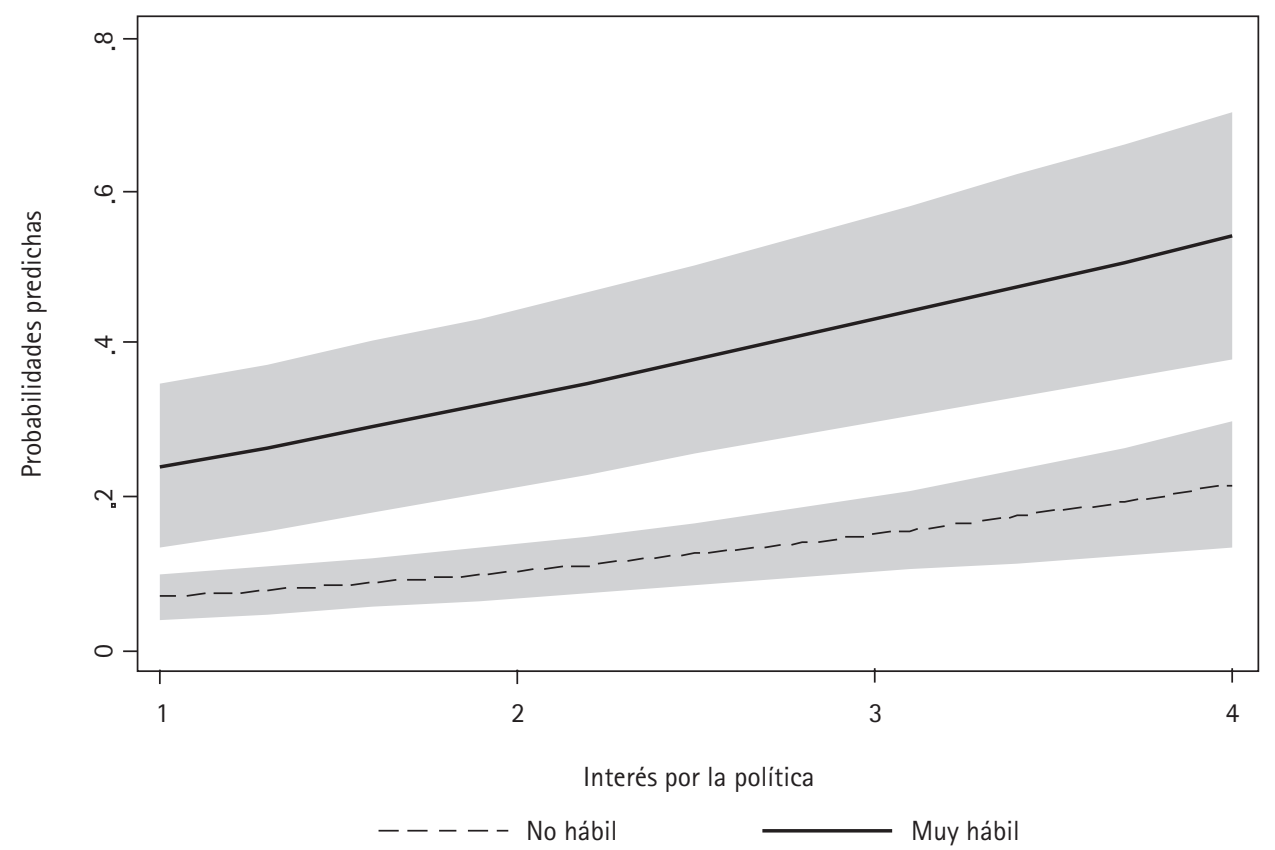

TABLA 5. REGRESIÓN MÚLTIPLE DE LAS HABILIDADES CON INTERNET POR EL RESTO DE VARIABLES EXPLICATIVAS

\begin{tabular}{|l|c|c|}
\hline \multicolumn{3}{|c|}{ Habilidades con Internet (0-6) } \\
\hline Nivel educativo & $0.229^{* * *}$ & $(0.069)$ \\
Estudiante & $-0.382^{* * *}$ & $(0.108)$ \\
Trabajo doméstico no remunerado & -0.1435 & $(0.166)$ \\
Hombre & 0.1166 & $(0.073)$ \\
Ingresos & $0.1708^{* * *}$ & $(0.026)$ \\
Edad & $-0.026^{* * *}$ & $(0.004)$ \\
\hline Eficacia interna & $0.087^{*}$ & $(0.048)$ \\
Eficacia externa & $-0.07^{*}$ & $(0.039)$ \\
Confianza en instituciones políticas & 0.0078 & $(0.022)$ \\
Cocepción del deber ciudadano & -0.027 & $(0.022)$ \\
Concepción de la implicación de un buen ciudadano & -0.0139 & $(0.021)$ \\
\hline Información política por Internet & $0.2002^{* * *}$ & $(0.028)$ \\
Escuchar y ver las noticias (radio o TV) & -0.0536 & $(0.038)$ \\
Escuchar y ver otros programas sobre política (radio o TV) & $0.0458^{*}$ & $(0.030)$ \\
Leer el periódico (en papel o en Internet) & 0.0237 & $(0.025)$ \\
\hline Interés político & -0.019 & $(0.054)$ \\
\hline cons & $1.607^{* * *}$ & $(0.3)$ \\
\hline F & 20.67 & \\
Prob > F & 0.0000 & \\
R-cuadrada & 0.1740 & \\
N & 1772 & \\
\hline
\end{tabular}

Coeficientes de regresión y su significatividad: ${ }^{*} p<0.10, * * p<0.05, * * * p<0.001$. Errores estándar entre paréntesis. 
Los resultados muestran que el interés político no tiene impacto significativo en el desarrollo de las habilidades con Internet, por lo que podemos descartar la idea de que los más interesados en política sean también los más habilidosos internautas. Por tanto, la relación entre las habilidades con Internet y la participación no es espuria y debida realmente al interés por la política. En este sentido encontramos resultados diferentes a los hallados por los autores anteriormente mencionados, y que fueron comentados en la segunda sección del artículo. Las habilidades por Internet son independientes del interés por la política.

Finalmente, respecto a la segunda hipótesis, en la que estableciamos que navegar por Internet sin objetivo concreto y ser contactado via email tendría un efecto directo sobre la participación online, los resultados de la tabla 4 confirman que estas dos variables tienen un efecto en la participación, tanto en relación con el número de actividades realizadas (regresión múltiple) como en el caso de llevar a cabo al menos una actividad (regresión logística). Estas variables fueron propuestas como posibles mecanismos causales que podian explicar cómo los internautas habilidosos sin especial interés por la política acababan participando en línea. Estos usuarios frecuentes y habilido-

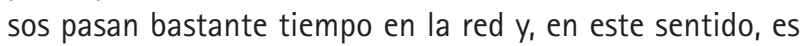
muy probable que estén expuestos a peticiones inesperadas para participar en línea, sobre todo si también navegan a menudo por Internet sin un objetivo concreto.

\section{Conclusión}

El análisis de estos datos no corrobora la tesis más radical que dice que el uso de Internet elimina el efecto de la motivación en la participación (DiGenaro y Dutton, 2006), pero sí está en línea con la tesis más moderada que dice que el uso de Internet tiene un efecto directo en la participación independientemente de la motivación (Xenos y Moy, 2007). Estos resultados no permiten concluir que la motivación haya dejado de ser un factor importante para explicar los diferentes niveles observados de participación online. De hecho, la propensión a participar entre usuarios de Internet, tanto experimentados como no experimentados, siempre aumenta con la motivación. Lo que si permiten concluir estos resultados es que basta con ser un usuario experimentado de Internet para que, independientemente de la motivación, aumente la probabilidad de participar en política dentro de la red.

En consecuencia, se confirma nuestra primera hipótesis, que establecía que disponer de habilidades con Internet no haría desaparecer el efecto del interés político sobre la participación, pero que sí tiene un efecto directo sobre la participación, independiente del interés por la política. Internet, por tanto, está teniendo un efecto sobre la implicación en actividades políticas que va más allá de las teorias del refuerzo de los perfiles de participantes ya existentes en el mundo presencial. Se puede considerar que un nuevo perfil está comenzando a realizar, al menos, una actividad en política: Ios usuarios habilidosos con Internet que carecen de motivación por la política.

Respecto a la segunda hipótesis, se determinaron dos posibles mecanismos que podían dar cuenta de la participación en línea de los usuarios frecuentes de Internet que no estaban interesados en política: navegar sin objetivo específico y ser contactado por correo electrónico. Los resultados indican que ambos factores producen un efecto directo sobre la participación online y, por tanto, deben ser tenidos en cuenta para comprender el proceso de participación en línea de personas no motivadas políticamente pero que pasan mucho tiempo en la red y dominan las herramientas y las oportunidades que ofrece Internet.

En resumen, podemos concluir, junto con otros autores como Best y Krueger (2005), Gibson, Lusoli y Ward (2005) y Anduiza, Gallego y Cantijoch (2010), que los nuevos recursos online, o lo que denominamos habilidades con Internet, deben ser incluidos en el modelo explicativo clásico de la participación, al menos si se quiere explicar la participación online. Pero, además, este modelo explicativo tradicional debería refinarse y adaptarse para poder aceptar el hecho de que, para los internautas experimentados que participan online, el interés por la política no es tan relevante. Este fenómeno abre toda una serie de interrogantes sobre cuál será el papel de la motivación política en un mundo en el que Internet esté ampliamente extendido y también sea uno de los medios más importantes para la participación política y la acción colectiva. 
1 Ver, por ejemplo, Krueger (2002); Best y Krueger (2005); Anduiza, Gallego y Cantijoch (2010) y Cantijoch (2009).

2 Di Genaro y Dutton (2006) y Xenos y Moy (2007).

3 Para un buen resumen sobre estas posiciones, ver Boulianne (2009).

4 Esto puede deberse a varias razones. Una es que Internet efectivamente tenga un efecto movilizador y que éste solo se haya hecho visible por efecto del paso del tiempo. Otra es que haya un sesgo de selección y que sólo vean la luz, como apunta Boulianne (2009: 195), aquellos estudios que encuentran efectos positivos del uso de Internet sobre la participación. En este último caso, se está sobreestimando el efecto positivo del uso de Internet en la participación.

5 En otros estudios, como el de Weber, Loumakis y Bergman (2003), en los que no se controla el efecto del interés político y que muestran un efecto positivo del uso de Internet en la participación, queda la duda de qué pasaría si se controlara esta variable.

6 Traducción propia.

7 Traducción propia.

8 Este argumento es rebatido por Krueger (2006). En un esplendido artículo, este autor se dedica a exponer las barreras que existen para utilizar Internet con fines de movilización política por parte de las organizaciones políticas.

9 Esta encuesta fue diseñada por el equipo POLNET de la UAB y forma parte del proyecto SEJ2007-60082 financiado por el Ministerio Español de Ciencia e Innovación. El tamaño

Recibido: 1 de octubre de 2011 Aceptado: 20 de febrero de 2012 para un nivel de confianza del $95,5 \%$. El procedimiento muestral ha sido el comúnmente empleado por el CIS: polietápico, estratificado por conglomerados de población y selección de individuos por rutas aleatorias y cuotas de sexo y edad. El número del estudio del CIS es 2736. El cuestionario está disponible en línea: http://www. polnetuab.net/result.php?pagina $=$ Dat os\&ldioma $=E s p a \% F 10 \mid \& j p g=03$.

10 Cantijoch (2009); Anduiza, Gallego y Jorba (2009); Anduiza, Gallego y Cantijoch (2010); Anduiza, Cantijoch, Gallego y Salcedo (2010); Anduiza, Cantijoch, Colombo, Gallego y Salcedo (2010).

11 Para una descripción de la situación de la participación online en España (frecuencias, modos de participación, perfiles de participantes, algunas explicaciones) y de los principales datos de la encuesta, consultar el monográfico de Anduiza, Cantijoch, Gallego y Salcedo (2010) y un artículo más breve con los primeros resultados de Anduiza, Cantijoch, Colombo, Gallego y Salcedo (2010).

12 El coeficiente de fiabilidad de KuderRichardson equivale a 0,6307 y el análisis de componentes principales muestra que las cinco actividades tienen un peso importante en el primer componente (por encima de $0,30)$.

13 Consideramos que una persona habilidosa con Internet es la que lleva a cabo varias y diversas actividades en línea. Aunque en el cuestionario existen hasta nueve actividades hemos seleccionado las seis que están más asociadas entre ellas según los resultados del coeficiente de fiabilidad Kuder-Richardson -0,555- y del análisis de componentes principales que muestra que las seis actividades seleccionadas tienen un peso impor- 
tante (por encima de 0,30) en el primer componente. Las seis actividades online seleccionadas son: comprar algún producto o servicio (alimentación, libros, cine, viajes, etc.); realizar gestiones bancarias; recibir o enviar mensajes de correo electrónico; Ilamar a través de Internet (skype, etc.); descargar archivos (documentos, música, vídeo, software, etc.) y mantener tu propio blog o página web.

14 En todo caso, los esfuerzos movilizadores forman parte del modelo clásico de los recursos para la participación, el cual se compone de tres elementos clave: recursos, implicación psicológica con la política y redes de "reclutamiento" (Verba, Scholzman y Brady, 1995: 269). La novedad aquí es que esta llamada a la acción política se realiza a través de un nuevo medio que es Internet, y que nuestra hipótesis, contraria a la que defienden Verba, Scholzman y Brady (1995: 270), plantea que, en ausencia de implicación psicológica, la participación se puede producir en el caso de las actividades en línea.

15 Para calcular las probabilidades y poder realizar los gráficos, los valores de las variables explicativas que actúan como posibles mecanismos causales (ser contactado por email, navegar por Internet sin rumbo fijo) se han fijado a cero, asi como otras dos variables dicotómicas (ser estudiante y dedicarse a un trabajo doméstico no remunerado). La variable dicotómica ser hombre se ha fijado a 1. Las variables continuas se han fijado en sus medias.

16 Se han excluido del análisis navegar por Internet sin rumbo fijo y ser contactado a través del correo electrónico por temas políticos porque éstos se proponen como mecanismos causales en la relación entre habilidades con Internet y participación política en línea; es decir, no pueden considerarse en ningún caso como variables causales de las habilidades con Internet porque aquí se plantean como consecuencias. En cambio, se ha optado por dejar en el modelo la variable obtener información política a través de Internet, que también podría imaginarse como una consecuencia más que como una causa de las habilidades con Internet, pero que en este artículo no la hemos planteado como mecanismo causal entre las habilidades con Internet y la participación. Mantener esta variable en el modelo, sin embargo, plantea un problema adicional y es que, dado que está muy correlacionada tanto con las habilidades con Internet como con el interés político, puede estar capturando el efecto positivo del interés por la política sobre las habilidades con Internet. Para descartar esta posibilidad se ha ejecutado el modelo sin esta variable y el resultado ha sido el mismo: el interés por la política sigue sin tener un efecto positivo significativo en las habilidades con Internet.

\section{BIBLIOGRAFÍA}

Aldrich, J. H. (1983): "A Downsian Spatial Model with Party Activism", The American Political Science Review 77, pp. 974-990.

Anduiza, E.; Cantijoch, M. y Cristancho, C. (2009): "Los ciudadanos y el uso de Internet en la campaña electoral", en Montero, J. R. y Lago, I. (eds.), Las elecciones generales de 2008. Madrid, CIS.

Anduiza, E.; Cantijoch, M. y Gallego, A. (2009): "Political Participation and the
Internet", Information, Communication and Society, 12: 6, pp. 860-878.

Anduiza, E.; Cantijoch, M.; Colombo, C.; Gallego, A. y Salcedo, J. (2010): "Los usos políticos de Internet en España", Revista Española de Investigaciones Sociológicas 129, pp. 133-146.

Anduiza, E.; Cantijoch, M.; Gallego, A. y Salcedo, J. (2010): Internet y participación política en España, Colección Opiniones y Actitudes, núm. 63. Madrid, CIS.

Anduiza, E.; Gallego, A. y Cantijoch, M. (2010): "Online Political participation in Spain: The Impact of Traditional and Internet Resources", Journal of Information, Technology and Politics, 7 (4).

Anduiza, E.; Gallego, A. y Jorba, L. (2009): "The Political Kowledge Gap in the New Media Environment: Evidence from Spain", Paper presentado en las Joint Sessions of the ECPR, Lisboa.

Barry, B. (1978): Sociologists, Economists and Democracy, Chicago, The University of Chicago Press.

Best, S. J. y Krueger, B. S. (2005): "Analysing the Representativeness of Internet Political Participation", Political Behavior 27 (2), pp. 183-215.

Bimber, B. (2001): "Information and Political Engagement in America: The Search for Effects of Information Technology at the Individual Level", Political Research Quaterly. 53 (1), pp. 53-67.

Bimber, B. (2003): Information and American Democracy, New York, Cambridge University Press.

Boncheck, M. S. (1995): "Grassroots in Cyberspace: Recruiting Members on the Internet or Do Computer Networks Facilitate Collective Action? A Transaction Cost Approach", 53rd Annual Meeting of the Midwest Political Science Association, Chicago.

Boulianne, S. (2009): "Does Internet Use Affect Engagement? A Meta-Analysis 
of Research", Political Communication, 26, pp. 193-211.

Cantijoch, M. (2009): "Reinforcement and mobilisation: the influence of the Internet on different types of political participation", International Seminar "Citizen Politics: Are the New Media Reshaping Political Engagement'", Barcelona, UAB.

Clark, P. B. y Wilson, J. O. (1961): "Incentive Systems: A Theory of Organizations", Administrative Science Quarterly, 6, pp. 129-166.

Delli Carpini (2000): "Gen.com: Youth, Civic Engagement, and the New Information Environment", Political Communication, 17 (4), pp. 341-349.

Downs, A. (1957): An Economic Theory of Democracy, New York, Harper y Row.

Di Genaro, C. y Dutton, W. (2006): "The Internet and the public: Online and offline political participation in the UK", Parliamentary Affairs, 59, pp. 299-313.

Gibson, R. K.; Lusoli, W. y Ward, S. (2005): "Online participation in the UK: Testing a 'Contextualised' Model of Internet Effects", British Journal of Politics and International Relations, 7, pp. 561-583.

Granick, S. (2005): "A Reconceptualization of the Antecedents of Party Activism: A Multidisciplinary Approach", Political Studies 53, pp. 598-620.

Jensen, M. J.; Danzinger, J. N. y Venkatesh, A. (2007): "Civil Society and Cyber Society: The Role of the Internet in Community Associations and Democratic Politics", The Infomation Society, 23, pp. 39-50.
Karakaya, R. (2005): "The Internet and Political Participation. Exploring the Explanatory Links", European Journal of Communication, 20, pp. 435559.

Krueger, B. (2002): "Assesing the Potential of Internet Political Participation in the United States. A Resource Approach", American Political Research, Vol. 30 (5), pp. 476-498.

Krueger, B. (2006): "A Comparison of Conventional and Internet Political Mobilization", American Politics Research, 34, pp. 759-776.

Margolis, M. y Resnick, D. (2000): Politics as Usual: The "Cyberspace Revolution", Thousand Oaks, CA: Sage Publications.

Mossberger, K. (2009): "Toward digital citizenship: addresing inequality in the information age", en Chadwick, A. H. y Howard, P. N. (Ed.), Routledge Handbook of Internet Politics. London, Routledge.

Mossberger, K.; Tolbert, C. J. y McNeal, R. S. (2008): Digital Citizenship. The Internet, Society, and Participation, Cambridge, Massachusetts, The MIT Press.

Norris, P. (2001): Digital divide: Civic engagement, information poverty and the Internet worldwide, Cambridge, United Kingdom:, Cambridge University Press.

Olson, M. (1965): The Logic of Collective Action. Public Goods and the Theory of Groups, Cambridge, Massachusetts, Hravard University Press.

Putnam, R. (2000): Bowling Alone, New York: Simon and Schuster.
Quintelier, E. y Vissers, S. (2008): "The Effect of Internet Use on Political Participation. An Analysis of Survey Results for 16-Year-Olds in Belgium", Social Science Computer Review, 26, pp. 411-427.

Riker, W. y Ordeshook, P. C. (1968): "A theory of the calculus of voting", American Political Science Review, 61, pp. 25-41.

Tolbert, C. y McNeal, R. (2003): "Unraveling the Effects of the Internet on Political Participation?", Political Research Quaterly, 56 (2), pp. 175-185.

Verba, S.; Schlozman, K. L. y Brady, H. E. (1995): Voice and Equality. Civic Voluntarism in American Politics, Cambridge, Harvard University Press.

Ward, S.; Gibson, R. K. y Lusoli, W. (2003): "Participation and Mobilization online: Hype, hope and reality", Parliamentary Affairs, 56, pp. 652-668.

Weber, L. M.; Loumakis, A. y Bergman, J. (2003): "Who participates and why? An analysis of citizens on the Internet and the mass public", Social Science Computer Review, 21, pp. 26-42.

Whiteley, P. y Seyd, P. (1998) The Dynamics of Party Activism in Britain: A Spiral of Demobilization British Journal of Political Science, 28, pp. 113-137.

Whiteley, P.; Seyd, P.; Richardson, J. y Bissell, P. (1994): "Explaining Party Activism: The Case of the British Conservative Party", British Journal of Political Science, 24, pp. 79-94.

Xenos, M. y Moy, P. (2007): "Direct and Differential Effects of the Internet on Political and Civic Engagement", Journal of Communication, 57, pp. 704-718. 